www.nature.com/bmt

\title{
Corrigendum
}

\section{Defibrotide in the treatment of children with veno-occlusive disease (VOD): a retrospective multicentre study demonstrates therapeutic efficacy upon early intervention}

S Corbacioglu, J Greil, C Peters, N Wulffraat, HJ Laws, D Dilloo, B Straham, U Gross-Wieltsch, KW Sykora, A Ridolfi-Luthy, O Basu, B Gruhn, T Gungor, W Mihatsch ${ }^{1}$ and AS Schulz

\section{Bone Marrow Transplantation (2004) 33, 673. doi:10.1038/sj.bmt.1704494}

Correction to: Bone Marrow Transplantation (2004) 33, 189-195. doi:10.1038/sj.bmt.1704329; Published online: 8 December 2003.

The author names and affiliations were published incorrectly; the correct version is as follows:

S Corbacioglu ${ }^{1}$, J Greil ${ }^{2}$, C Peters ${ }^{3}$, N Wulffraat ${ }^{4}$, HJ Laws $^{5}$, D Dilloo 5 , B Strahm ${ }^{6}$, U Gross-Wieltsch ${ }^{7}$, KW Sykora $^{8}$, A Ridolfi-Lüthy ${ }^{9}$, O Basu ${ }^{10}$, B Gruhn ${ }^{11}$, T Güngör ${ }^{12}$, W Mihatsch ${ }^{1}$ and AS Schulz ${ }^{1}$

\begin{abstract}
${ }^{1}$ Universitätsklinik und Poliklinik für Kinder- und Jugendmedizin, Universität Ulm, Germany; ${ }^{2}$ Klinik für Kinderheilkunde und Jugendmedizin, Tübingen, Germany; ${ }^{3}$ St Anna Kinderspital, Vienna, Austria; ${ }^{4}$ Wilhelmina Children's Hospital, Utrecht, The Netherlands; ${ }^{5}$ University Children's Hospital, Düsseldorf, Germany; ${ }^{6}$ University Children's Hospital, Freiburg, Germany; ${ }^{7}$ Olga Hospital, Stuttgart, Germany; ${ }^{8}$ University Children's Hospital, Hannover, Germany; ${ }^{9}$ Inselspital, Bern, Switzerland; ${ }^{10}$ University Children's Hospital, Essen, Germany; ${ }^{11}$ Department of Pediatrics, University of Jena, Jena, Germany; and ${ }^{12}$ Kinderspital Zürich, Zürich, Switzerland
\end{abstract}

